\title{
Estudo da dinâmica da floresta manejada no projeto de manejo florestal comunitário do PC Pedro Peixoto na Amazônia Ocidental
}

\author{
Marcus Vinicio Neves d'OLIVEIRA', Evaldo Muñoz BRAZ² \\ RESUMO \\ Neste trabalho são apresentados resultados do estudo da dinâmica da floresta em um sistema de manejo florestal voltado para a \\ pequena propriedade. $\mathrm{O}$ sistema prescreve ciclo de corte de 10 anos e taxa de corte de $10 \mathrm{~m}^{3}$.ha ${ }^{-1} \mathrm{e}$ tração animal para o arraste. $\mathrm{O}$ \\ crescimento da floresta de $1 \mathrm{~m}^{3} \cdot$ ha $^{-1}$.ano-1 foi compatível com o ciclo e taxa de corte adotados. Os danos causados pela exploração \\ representaram $5 \%$ da área basal total, e a taxa de mortalidade quatro anos após a exploração foi 3,2 \%.
}

\section{PALAVRAS-CHAVE}

dinâmica de florestas, exploração florestal, manejo florestal

\section{Forest dynamics study of the managed forest of the PC Peixoto Communit forest management project in western Amazon}

\section{ABSTRACT}

On this work we present the results of a forest dynamics study performed in a forest management system designed for small properties. The system prescribes cutting cycles of 10 years, harvesting intensity of $10 \mathrm{~m}^{3} \mathrm{ha}^{-1}$ and animal traction for skidding. The forest growth was $1 \mathrm{~m}^{3} \mathrm{ha}^{-1}$ year-1 adequate to the proposed cutting cycle and harvesting intensity. The damage produced by the forest exploitation represented $5 \%$ of the total basal area of the forest and the mortality rate four years after exploitation was $3.2 \%$.

\section{KEY WORDS}

forest dynamics, forest exploitation, forest management

${ }^{1}$ Engenheiro Florestal PhD, pesquisador em manejo florestal. Embrapa Acre. Caixa Postal 321. Rio branco, Acre. e-mail: mvno@cpafac.embrapa.br

${ }^{2}$ Engenheiro florestal MSc, pesquisador em exploração florestal. Embrapa Acre. Caixa Postal 321. Rio Branco. Acre. e-mail:evaldo@cpafac.embrapa.br. 


\section{INTRODUÇÃO}

Como tradicionalmente ocorre em projetos de colonização na Amazônia, as florestas localizadas no Projeto de Colonização Pedro Peixoto no Acre, vêm sendo gradualmente substituídas por projetos de pecuária e agricultura desde a sua criação (Cavalcanti, 1994, Witcover et al., 1994). Este fato reflete uma tradição de uso da terra onde a floresta é vista como um obstáculo ao desenvolvimento e utilizada somente como uma fonte de recurso inicial (através da comercialização da madeira) para alavancar a instalação de projetos pecuários e lavouras de subsistência. Esta prática, com o tempo tende a atingir também as áreas de reserva legal, fato que já foi observado em projetos de colonização dos Estados do Acre e Rondônia (Valentin, 1989).

O sistema de manejo florestal estudado neste trabalho é uma alternativa para o uso das reservas legais para a produção sustentada de madeira e vem sendo aplicado no PC Peixoto desde 1996. Foi desenhado para pequenas propriedades de projetos de colonização ou assentamentos extrativistas e tem como objetivo gerar uma nova fonte de renda para estas famílias mantendo a estrutura e biodiversidade das florestas manejadas. Este trabalho teve por objetivo, estudar a dinâmica florestal (crescimento, ingresso, mortalidade e composição florística) nas áreas de floresta manejada do projeto de manejo florestal comunitário do PC Pedro Peixoto.

\section{MATERIAL E MÉTODOS}

\section{ÁREA DE ESTUDO}

O PC Peixoto foi criado em 1977, possui 317.588 ha, abrigando em torno de 3000 famílias de pequenos produtores rurais e está situado no Estado do Acre na Amazônia Ocidental (Figura 1). O clima é do tipo Aw (Köppen), em torno de três meses de período seco. A precipitação anual varia de $1.800 \mathrm{a}$ $2.000 \mathrm{~mm}$ e a temperatura média anual é de $24^{\circ} \mathrm{C}$. O período de estiagem vai de junho a setembro, permitindo que as atividades de exploração florestal sejam executadas até o início de outubro quando se iniciam as primeiras chuvas. Os solos predominantes são os Latossolos vermelho-amarelo, distróficos. A topografia predominante é plana, com declividade em torno de $5 \%$, e quase

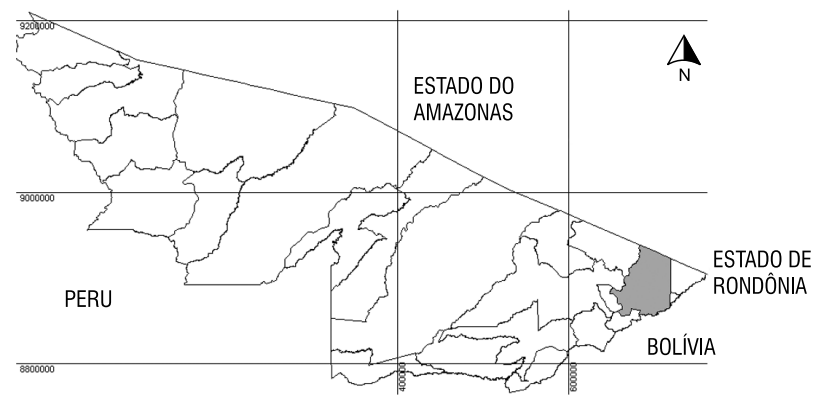

Figura 1- Mapa do Estado do Acre com o destaque da localização do PC Pedro Peixoto.

nunca superior a $10 \%$. A vegetação predominante é de floresta tropical semi-perenifólia, com formaçôes de floresta aberta e floresta densa. A parte não florestal, é basicamente formada por pastagens, culturas de subsistência e algumas culturas perenes (RADAMBRASIL, 1976).

A floresta manejada possui volume total médio (DAP $>10$ $\mathrm{cm}$ ) de 180,0 $\mathrm{m}^{3} \cdot \mathrm{ha}^{-1}$, com volume total de madeira de espécies com mercado para consumo interno e exportação, em torno de $42 \mathrm{~m}^{3} \cdot \mathrm{ha}^{-1}$, dos quais $20 \mathrm{~m}^{3}$.ha $\mathrm{h}^{-1}$ são de árvores com DAP acima de $50 \mathrm{~cm}$ (Oliveira et al., 1998).

\section{SISTEMA DE MANEJO FLORESTAL}

Cada um dos lotes do PC Pedro Peixoto tem em média 80,0 ha. O projeto de manejo florestal foi executado no fundo destes lotes, nas áreas de reserva legal, que possuem em torno de 40,0 ha. Os compartimentos (área explorada anualmente) foram dispostos de forma paralela a frente das propriedades. Cada um deles possuiu 4,0 ha ( $400 \mathrm{~m} \times 100 \mathrm{~m})$ (Figura 2).

O sistema foi desenhado para ser executado em um ciclo de corte mínimo de 10 anos, intensidade de corte entre 5 e 10 $\mathrm{m}^{3} \cdot \mathrm{ha}^{-1} \cdot$ ciclo $^{-1}$ e utilização de tração animal (bois) para o arraste da madeira através da floresta até as estradas secundárias. Tratamentos silviculturais (corte de cipós com um ano de antecedência) e técnicas de manejo florestal de impacto reduzido (microzoneamento, planejamento da exploração, seleção de espécies, corte direcionado das árvores) foram aplicadas à floresta.

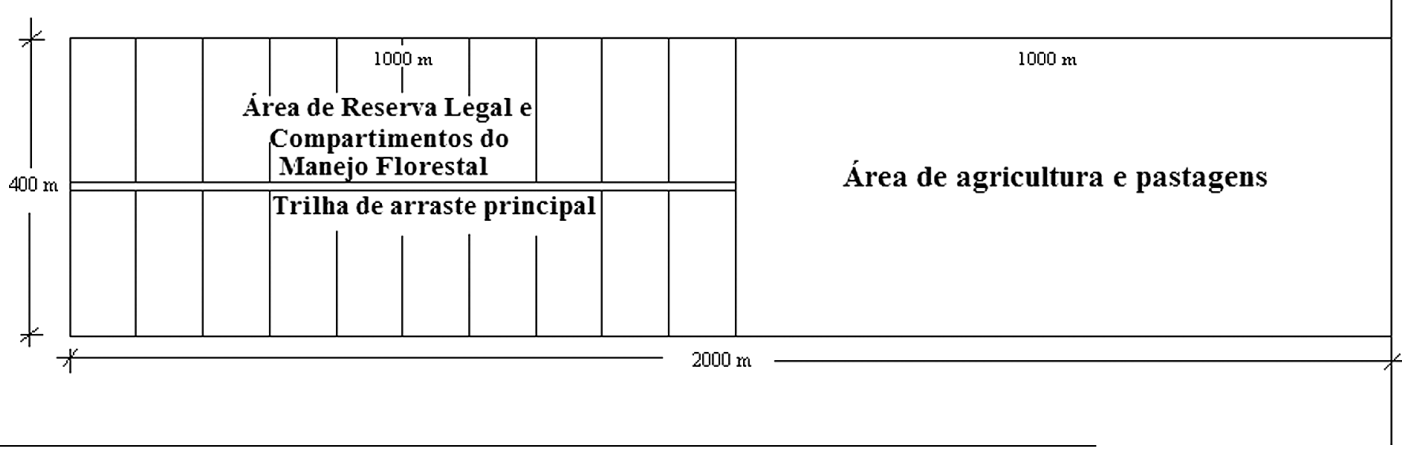

Figura 2 - Diagrama do lote e áreas de reserva legal no PC Pedro Peixoto. 
Para viabilizar o transporte primário não mecanizado, o processamento das toras foi feito com serraria portátil e motosserra, no próprio local onde as árvores foram cortadas (zona de abate) (Oliveira, 2000).

\section{ESTUDO DA DINÂMICA DA FLORESTA}

O estudo foi feito por meio de cinco parcelas permanentes alocadas dentro das áreas de manejo florestal avaliando crescimento, danos produzidos pela exploração, mortalidade, ingresso e composição florística. As parcelas possuem 1 ha (100 $\mathrm{m} \times 100 \mathrm{~m})$ subdivididas em 100 sub-parcelas com $100 \mathrm{~m}^{2}(10$ $\mathrm{x} 10 \mathrm{~m})$ cada. Todas as árvores com DAP e" $20 \mathrm{~cm}$ foram plaquetadas, identificadas e medidas. Em 20 sub-parcelas sorteadas também foram medidas e identificadas todas as árvores com DAP e" $5 \mathrm{~cm}$. O período de estudo foi de cinco anos. As árvores foram classificadas de acordo com a exposição de suas copas a luz solar (Silva et al., 1996) em três categorias:

1. Recebendo luz solar total na copa

2. Recebendo alguma luz solar direta na copa

3. Sem receber luz solar direta na copa

Foram considerados como ingresso todas as árvores com DAP e" $5 \mathrm{~cm}$. O cálculo da razão de ingresso foi padronizado como sendo a divisão do número total de plantas ingressantes em uma medição pelo número de adultos do censo anterior divididos pelo intervalo entre as duas medições (Condit et al., 1996). O incremento em diâmetro foi calculado de acordo com a fórmula: (dap2 - dap1) / t. Onde dap1 e dap2 são os diâmetros ao início e ao final do intervalo de medição t. A mortalidade média anual foi calculada de acordo com (Sheil et al., 1995): $\mathrm{M}=1-\left(\mathrm{N}_{1} /\right.$ $\mathrm{N}_{0}$ ) ${ }^{1 / \mathrm{t}}$, onde: $\mathrm{N}_{0}$ e $\mathrm{N}_{1}$ são a população no início e final do período de tempo $(\mathrm{t})$. Foram consideradas para o cálculo do dano produzido pela exploração todas as árvores quebradas, mortas ou danificadas pelo efeito das atividades ligadas a exploração da floresta. As espécies pioneiras foram utilizadas como indicadores das alterações na composição florística promovidas pela exploração.

\section{RESULTADOS E DISCUSSÃO}

3.1 Incremento em Área Basal e Danos Produzidos pela Exploração:

A área basal média nas parcelas permanentes antes da exploração foi de 22,51 $\mathrm{m}^{2}$.ha ${ }^{-1}$ dos quais $5,96 \mathrm{~m}^{2}$.ha $\mathrm{h}^{-1}$ de espécies comerciais. $\mathrm{O}$ corte das árvores causou a redução da área basal total para $20,88 \mathrm{~m}^{2}$.ha- $\mathrm{e}^{-1}$ das espécies comercias para 4,89 $\mathrm{m}^{2}$.ha1. Dois anos após a exploração florestal a área basal média total e de espécies comerciais, nestas parcelas, foi de respectivamente $21,12 \mathrm{~m}^{2} \cdot \mathrm{ha}^{-1}$ e $5,33 \mathrm{~m}^{2} \cdot \mathrm{ha}^{-1}$, o que representou um incremento médio anual neste período de $0,09 \mathrm{~m}^{2} \cdot \mathrm{ha}^{-1}\left(0,76 \mathrm{~m}^{3} \cdot \mathrm{ha}^{-1}\right)$ para a área basal total e $0,13 \mathrm{~m}^{2} \cdot \mathrm{ha}^{-1}\left(1,06 \mathrm{~m}^{3} \cdot \mathrm{ha}^{-1}\right)$ somente para as espécies comerciais. Quatro anos após a exploração, a área basal foi de $21,41 \mathrm{~m}^{2}$.ha-1. A diminuição do incremento em área basal neste período ocorreu devido a queda natural de árvores de grande porte dentro das parcelas neste período (Figura 3).

Os danos causados pela exploração representaram, em média, $1,21 \mathrm{~m}^{2} \cdot \mathrm{ha}^{-1}$ ou $5,1 \% \mathrm{da}$ área basal total das parcelas um ano após o corte. Os danos provocados por causas naturais (ventos ou tempestades), no mesmo período foram de $1,02 \mathrm{~m}^{2} . \mathrm{ha}^{-1}$, ou $4,3 \% \mathrm{da}$ área basal total. O dano à floresta residual causado pela exploração foi maior no primeiro ano após a exploração, provavelmente, pela morte de árvores danificadas durante a exploração (Maitre, 1987; Chai \& Sia, 1989; Primack et al., 1989; Abdul et al., 1992; Silva et al., 1996). Dois anos após a exploração, ainda haviam árvores morrendo como resultado da exploração, mas neste período, a mortalidade por causas naturais foi maior. Houve uma tendência do dano produzido por causas naturais aumentar após a exploração de $0,61 \mathrm{~m}^{2} \cdot \mathrm{ha}^{-1}$ antes $\mathrm{da}$ exploração para 1,61 $\mathrm{m}^{2} \cdot \mathrm{ha}^{-1}$ dois anos após (Figura 4).

\section{INCREMENTO EM DIÂMETRO}

$\mathrm{O}$ incremento em diâmetro variou de $2 \mathrm{~cm}^{-a n o^{-1}}$ para espécies pioneiras (ex. Jaracatea spinosa) a $0,1 \mathrm{~cm}$. ano $^{-1}$ para algumas espécies de sub-bosque (ex. Quaribea guianensis). A exposição das copas à luz do sol apresentou uma forte influência no incremento em diâmetro. Nas parcelas permanentes, esta variação foi de $0,57 \mathrm{~cm}$. ano ${ }^{-1}$ para árvores com copas completamente expostas à luz do sol a $0,28 \mathrm{~cm} . \mathrm{ano}^{-1}$ para plantas completamente sombreadas. Árvores que receberam alguma luz solar sobre a copa apresentaram incremento médio anual em diâmetro de 0,49 $\mathrm{cm}$ (Figura 5). Os resultados de incremento em diâmetro encontrados neste trabalho foram semelhantes a resultados obtidos em outros trabalhos realizados em florestas tropicais (e.g. Okali \& Ola-Adams 1988; Chiew \& Garcia 1989; Primack et al., 1989; Silva et al., 1996). O efeito da exposição das copas no incremento em diâmetro também é bem conhecido (e.g. Silva $e t$ al., 1995).

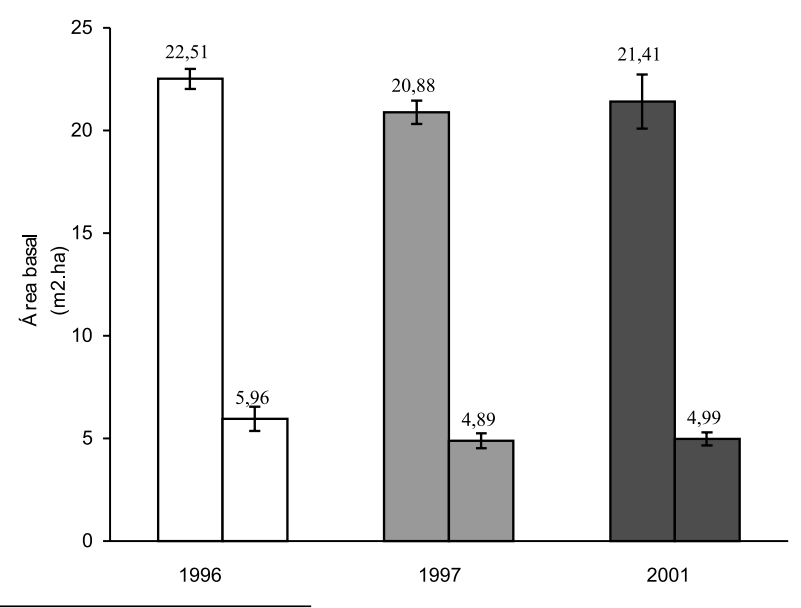

Figura 3 - Área basal ( $\mathrm{m}^{2}$.ha) total e de espécies comerciais, um ano antes (1996), imediatamente após (1997) e quatro anos depois da exploração (2001). Barras representam erro padrão $(p<0,05)$. 


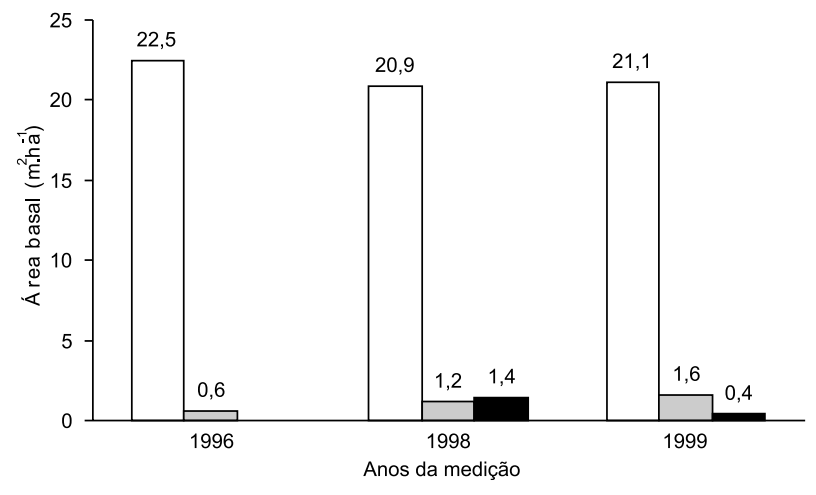

Figura 4 - Área basal ( $\mathrm{m}^{2}$.ha), aproveitável (colunas brancas), danificada por causas naturais (colunas cinza) e danificada pela exploração (colunas pretas), um ano antes (1996), um (1998) e dois (1999) anos após a exploração.

No entanto, os resultados apresentados neste trabalho demonstram que um considerável aumento no incremento (em torno de $100 \%$ ) anual em diâmetro pode ser esperado após uma mudança na exposição de copa de uma árvore. Este resultado é um forte argumento para a aplicação de tratamentos silviculturais de abertura de copagem na região, considerando que apenas uma pequena percentagem das árvores da floresta possuem copas totalmente expostas a luz solar e que a maioria delas estão totalmente sombreadas (Figura 6).

\section{INGRESSO DE NOVAS PLANTAS}

A população de árvores na classe de DAP de 5 a $10 \mathrm{~cm}$ diminuiu após a exploração. $\mathrm{O}$ ingresso verificado no período ainda não foi suficiente para atingir o número de plantas de

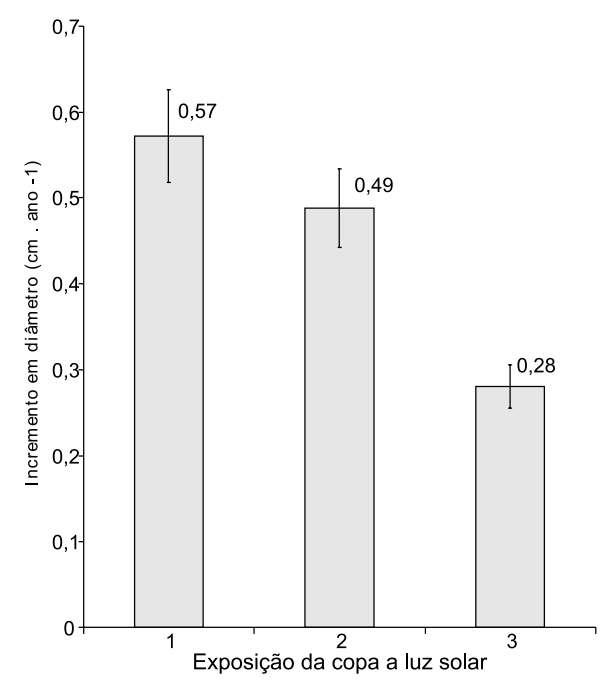

Figura 5 - Incremento médio, em diâmetro, nos quatro primeiros anos após a exploração de acordo com a exposição da copa: 1- luz total na copa; 2 alguma luz direta sobre a copa; 3 - sem receber luz direta. Barras representam erro padrão $(p<0,05)$. antes da exploração. Como a população de plantas nesta classe de DAP vem decrescendo com o tempo, podemos concluir que a taxa de mortalidade ainda é superior a taxa de ingresso. Parte disto se deve ao baixo nível de impacto na floresta causado pelo manejo, com a remoção de poucas árvores e a não utilização de mecanização, restringindo a entrada de espécies pioneiras. Também o período coberto pelo estudo não foi suficiente para permitir que a maioria das espécies potencialmente ingressantes nas clareiras atingissem o DAP mínimo considerado neste estudo $(5 \mathrm{~cm})$ (Figura 7).

Embora o ingresso de árvores de espécies comerciais no período do estudo tenha sido pequeno, foi suficiente para manter a população destas espécies no mesmo patamar de antes da exploração. Isto pode ser interpretado como tendo sido o banco de mudas destas espécies existente antes da exploração, suficiente para suportar o impacto da exploração florestal (Figura 8). Caso

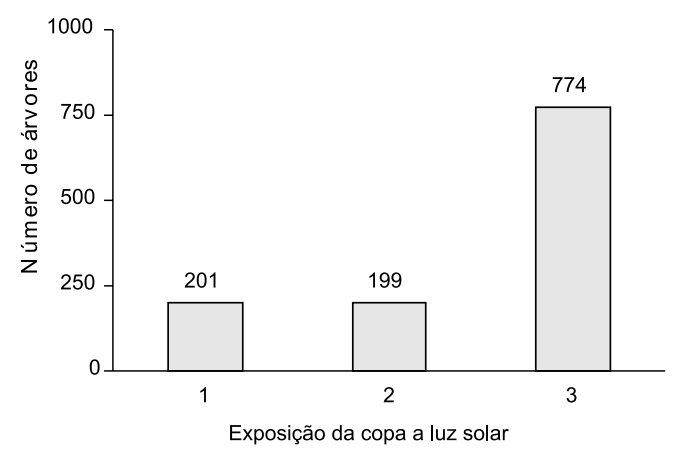

Figura 6 - Distribuição das árvores nas parcelas permanentes do PC Peixoto de acordo com a iluminação de copa: 1 - luz total na copa; 2 alguma luz direta na copa; 3 não recebe luz direta na copa.

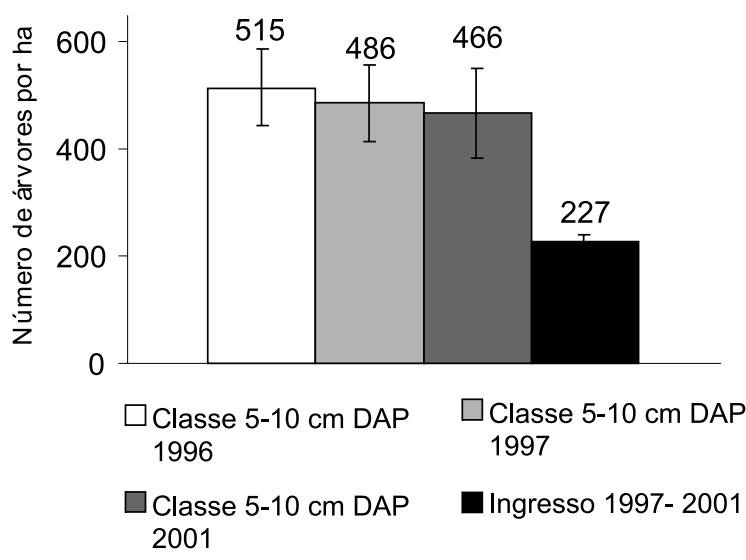

Figura 7 - Número de árvores na classe de 5-10 cm de DAP, um ano antes, imediatamente e quatro anos após a exploração nas PP do PC Peixoto e ingresso de novas plantas no período de 4 anos (barras representam 0 erro padrão da média $p<0,05)$. 
nas próximas medições a taxa de ingresso continue crescendo, então poderemos afirmar que as árvores matrizes deixadas estão cumprindo com a sua função de regenerar a floresta.

\section{MORTALIDADE}

A taxa de mortalidade média de árvores nos quatro primeiros anos após a exploração foi de $3,2 \%$. As pequenas variaçôes desta taxa nas diferentes classes de diâmetro não permitiu identificar maior ou menor mortalidade em nenhuma classe de diâmetro. A elevada taxa de mortalidade na classe de diâmetro de 80,0 $89,9 \mathrm{~cm}$ é reflexo da baixa população destas árvores, e foi provocada pela morte de uma única árvore no período do estudo (Figura 9).

\section{RIQUEZA DE ESPÉCIES E COMPOSIÇÃO FLORÍSTICA}

A exploração florestal conduzida no PC Peixoto não resultou em um aumento significativo da população de espécies pioneiras, como normalmente ocorre quando a exploração florestal é mecanizada (Figura 10). A população de espécies comerciais exploradas também não foi afetada de forma significativa pelo manejo da floresta (Figura 11).

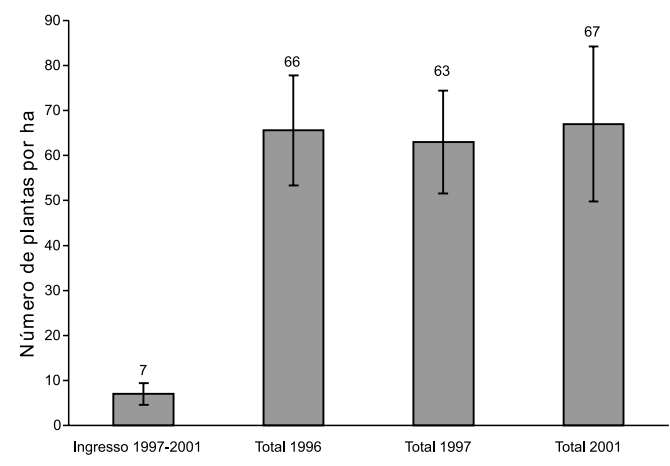

Figura 8 - Ingresso e número total da plantas de espécies comerciais na classe de 5,0 - 9,9 cm de DAP, antes da exploração (1996), um ano após a exploração (1997) e quatro anos após a exploração. Barras representam erro padrão $(p<0,05)$.

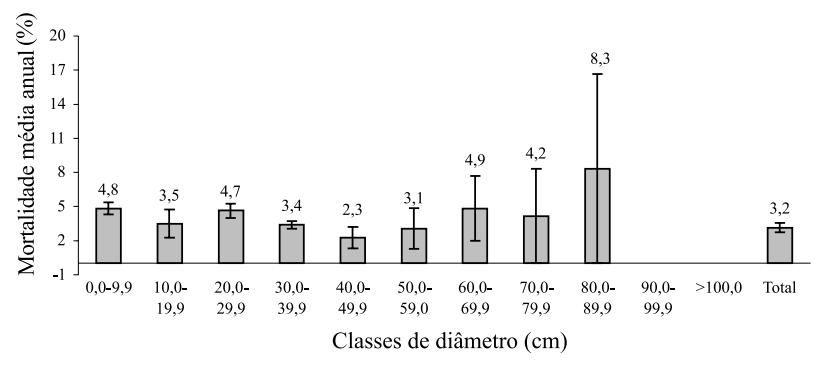

Figura 9 - Mortalidade média anual (\%), por classe de DAP e considerando todas as árvores amostradas (Total), na floresta manejada nos quatro primeiros anos após a exploração. Barras representam erro padrão $(p<0,05)$.

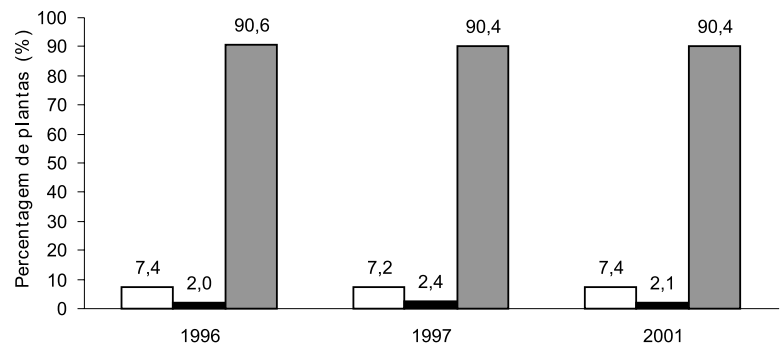

Figura 10 - Distribuição percentual das plantas nas parcelas permanentes do PC Peixoto, no grupo das palmeiras (colunas brancas), das espécies pioneiras (colunas pretas), não pioneiras (colunas cinza), um ano antes (1996), imediatamente depois (1997) e quatro anos (2001) após a exploração.

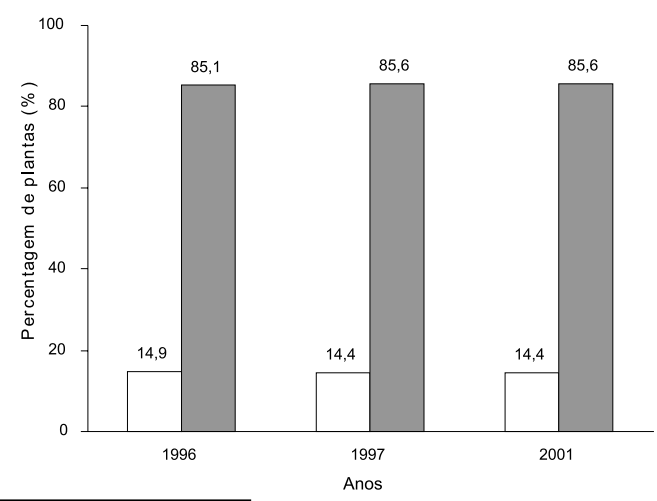

Figura 11 - Distribuição percentual das plantas nas Parcelas Permanentes do PC Peixoto das espécies comerciais (colunas brancas) e não comerciais (colunas cinza), um ano antes (1996), imediatamente após (1997) e quatro anos após (2001) a exploração.

\section{CONCLUSÃO}

O crescimento das árvores remanescentes das espécies comerciais, nos primeiros quatro anos após a exploração, foi compatível com o ciclo de corte e intensidade de corte utilizados, o que deverá possibilitar a recomposição da floresta para o próximo corte.

O tamanho das clareiras produzidas pela exploração não foram suficientes para promover o aumento da população de espécies pioneiras de ciclo curto nas área de manejo contribuindo para a diminuição da competição para o estabelecimento das espécies comerciais e potenciais na regeneração natural.

Apesar de ainda ser muito cedo para afirmar que as árvores matrizes serão suficientes para garantir a manutenção da população das espécies manejadas, as mudas destas espécies estocadas na floresta residual, foram suficientes para recompor esta população em quatro anos, ao mesmo nível existente antes da exploração da floresta.

O incremento em diâmetro diferenciado das árvores de acordo com a exposição das copas a luz solar e o grande número de plantas sob sombra encontrados na floresta, são fortes argumentos 
em favor da aplicação de tratamentos silviculturais para promover a liberação da competição por luz das copas nas áreas de manejo, como forma de aumentar a produtividade da floresta .

\section{BIBLIOGRAFIA CITADA}

Abdul, K.R.; Wan Razali, W.M.; Shahrulzaman, I.; Azman, H. 1992. Growth response of hill dipterocarp forest following two methods of logging in Peninsular Malaysia. In: Proceedings of the Symposium on Harvesting and Silviculture for Sustainable Forestry in the Tropics. Kuala Lumpur, Malaysia, p. 24-31.

Cavalcanti, T.J.S. 1994. Colonização no Acre: uma análise socioeconômica do projeto de assentamento dirigido Pedro Peixoto. Tese de Mestrado. Universidade Federal do Ceará - Centro de Ciências Agrárias. Fortaleza, Brasil. 196pp.

Chai, F.Y.C.; Sia P.C. 1989. Stand table projections for a mixed swamp forest of Sarawak. In: Proceedings of the Seminar on Growth and Yield in Tropical Mixed/moist Forest. Forest Research Institute, Malaysia, p. 60- 77.

Chiew, K.Y.; Garcia, A. 1989. Growth and yield studies in the Yayasan Sabah forest concession area. In: Mohd, W.R.W.; Chan, H.T.; Appanah, S. (Eds.) Proceedings of the Seminar on Growth and Yield in Tropical Mixed/moist Forest. Forest Research Institute, Malaysia, pp. 192-205.

Condit, R.; Hubbell, S.P.; Lafrankie, J.V.; Sukumar, R.; Manokaran, N.; Foster, R.B.F.; Ashton, P.S. 1996. Species-area and speciesindividual relationships for tropical trees: a comparison of three 50-ha plots. Journal of Ecology, 84: 549-562.

Maitre, H. F. 1987. Natural forest management in Cote d'Ivoire. Unasylva, 39(3-4): 53-60.

Okali, D.U.U.; Ola-Adams, B.A. 1988. The population changes in treated rainforest at Omo Forest Reserve, south-western Nigeria. Journal of Tropical Ecology, 3: 291-313.

Oliveira, M.V.N.d'; Braz, E.M.; Burslem, D.F.R.P.; Swaine, M.D. 1998. Small-Scale Natural Forest Management: A new model for small farmers in the Brazilian Amazon. Tropical Forest Update, $8(1): 5-7$.

Oliveira, M.V.N. d' . 2000. Sustainable Forest Management for Small Farmers in Acre State in the Brazilian Amazon. PhD thesis (unpublish) Aberdeen University, Plant and Soil Science Department. 167pp.
Primack, R.B.; Chai, E.O.K.; Tan, S.S.; Lee, H.S. 1989. Relative performance of dipterocarp trees in natural forest, managed forest, logged forest and plantations throughout Sarawak, East Malaysia. In: Mohd, W.R.W.; Chan, H.T.; Appanah, S. (Eds.) Proceedings of the Seminar on Growth and Yield in Tropical Mixedl Moist Forest. Forest Research Institute, Malaysia. p. 161-175.

Radambrasil. 1976. Levantamento dos Recursos Naturais. Folha SC19, Rio Branco. Vol. 12, DNPM, MME. Rio de Janeiro, Brasil, 458pp.

Sheil, D.; Burslem, D.F.R.P.; Alder, D. 1995. The interpretation and misinterpretation of mortality rate measures. Journal of Ecology, 83: 331-333.

Silva, J.N.M.; Carvalho, J.O.P.; Lopes.J.C.A.; Almeida, B.F.; Costa, D.H.M.; Oliveira, L.C.; Vanclay, J.K.; Skovsgaard, J.P. 1995. Growth and yield of a tropical rain forest in the Brazilian Amazon 13 years after logging. Forest Ecology and Management, 71: 3, 267-274.

Silva, J.N.M.; Carvalho, J.O.P.; Lopes, J.C.A.; Oliveira, R.P.; Oliveira, L.C. 1996. Growth and yield studies in the Tapajos region, Central Brazilian Amazon. Commonwealth Forestry Review, 75(4): 325-329.

Valentim, J.F. 1989. Research indicates technology to avoid deforestation and burning for cattle ranching activities in the state of Acre, Brazilian Amazon. Paper presented in the seminar 'Pasture improvement: a possible alternative to deforestation in the Amazon', 12 Oct. Agronomy Department, University of Florida, Gainesville, Fla. 5 pp.

Witcover, J.; Vosti, S.A.; Barbosa, F.R.A.; Batista, J.; Boklin G.; França, Sb; Castilla, C.; Fujisaka, S.; Gallo, S.L.F.; Garcia, J.H.; Cabral, W.G.; Hurtado, L.; Leite, A.; Leite, F.M.N.; Souza, D.A.; Marinho, J.T.S.; Rocha, K.; Neto, C.R. 1994. Alternatives to slash-and-burn agriculture (ASB): a characterisation of Brazilian benchmark sites of Pedro Peixoto and Theobroma August/September 1994. MP Working paper No. US 96-003. IFPRI, Washington, D C. 44 pp.

Recebido em 10/02/03 Aceito em 03/04/06 